\title{
Subkutane interne anteriore Fixation vs. Fixateur externe?
}

\section{Der Fixateur externe hat sich bisher in der Notfallbehandlung von Beckenringfrakturen etabliert. Aufgrund der möglichen Komplikationen verglichen Osterhoff et al. in vitro die mecha- nische Stabilität des Fixateur externe (Fix. ext.) mit einem sub- kutanen internen anterioren Fixateur (SIAF). Osterhoff $\mathrm{G}$ et al. Mechanical testing of a device for subcutaneous internal anterior pelvic ring fixation versus external pelvic ring fixation. BMC Musculoscelet Disord. 2014; $15: 111$}

\section{Einleitung \\ $\nabla$}

Neben den positiven Eigenschaften des Fixateur externe (Fix. ext.), wie die Reduktion des Blutverlustes und der Bewegung der Frakturfragmente sowie die Unterstützung der Hämostase in den ersten Tagen nach Trauma, ist die Verwendung auch mit möglichen Komplikationen verbunden. Diese beziehen sich hauptsächlich auf Pininfektionen, eingeschränkte Mobilisierbarkeit des Patienten, die Beeinträchtigung der operativen Zugangswege bei intraabdominalen Begleitverletzungen und die Gefährdung des abdominalen Weichteilgewebes bei stark adipösen Patienten. Mit der Entwicklung minimal-invasiver Instrumentierungssysteme entstanden neue Möglichkeiten, die Technik der subkutanen internen anterioren Beckenringfixation in vergleichbarer Zeit durchzuführen. Bislang ist wenig bekannt über die mechanischen Eigenschaften dieser Instrumentation.

\section{Material und Methode \\ $\nabla$}

Die Studie war als stufenweise Messung der Ermüdungszeit oder Beschädigungsakkumulation der Stab-Pin- bzw. StabSchrauben-Verbindungen aufgebaut. Die Fixateure wurden auf jeweils 2 Polyoxymethylen-Testzylindern verankert, die die Beckenknochen darstellten.

In der External-Gruppe wurde ein standardisierter Fix. ext. (Hoffmann II, Fa. Stryker) mit 2 gewindebohrenden Pins ( $5 \mathrm{~mm}$ Durchmesser) in Winkel und Abstand wie bei supraazetabulärer Anbrin- gung gewählt, mit 2 Karbonstangen durch Kopplungsstücke gemäß Herstellerangaben angebaut und über Drehmomentschraubenzieher befestigt.

In der SIAF Gruppe wurden 2 Gewindeschrauben (Iliac Multiaxial Screw, Fa. Medtronic, $8 \mathrm{~mm}$ Durchmesser) platziert und die polyaxialen Schraubenköpfe durch einen Titan-Stab verbunden. Es wurden jeweils 3 Tests pro Gruppe mit 2000 fortlaufenden zyklischen Belastungen durch eine universale Testmaschine (ElektroPulse, Fa. Instron) mit einer Frequenz von $1 \mathrm{~Hz}$ unter sinusförmiger lateraler Kompression und Distraktion $( \pm 50$ $\mathrm{N})$ sowie Rotation $( \pm 0,5 \mathrm{Nm})$ durchgeführt, wobei die Belastung alle 200 Zyklen alternierte. Während des Tests wurden Belastungskraft und Dislokation registriert und durch eine Kraftmessdose sowohl die Translationssteifigkeit als auch die Rotationssteifigkeit nach 100, 300, 500, 700 und 900 Zyklen berechnet und mittels T-Test mit SPSS.

\section{Ergebnisse}

Es gab keinen signifikanten Unterschied für die Translationssteifigkeit zwischen dem SIAF und dem Fix. ext. für die direkten Vergleiche bei $500(p=0,089), 700$ $(\mathrm{p}=0,081)$ und $900(\mathrm{p}=0,266)$ Zyklen. Die Rotationssteifigkeit allerdings fiel beim SIAF bei $300(p=0,005), 500(p=0,020)$ und $900(p=0,005)$ Zyklen um 50\% höher aus als beim Fix. ext. In allen Versuchen wurden weder Lockerungen noch sonstige Schädigungen unter der applizierten Belastung beobachtet.

\section{Diskussion \\ $\nabla$}

Die Methode eines subkutanen Beckenringfixateurs wurde erstmals von Kuttner et al. 2009 beschrieben. Da so der Drehdurchmesser des Implantats über den Beckenknochen gesenkt und durch die subkutane Konstruktion die Häufigkeit von Komplikationen wie Pininfektionen und -lockerungen vermindert werden konnten, wurde der SIAF als alternative Methode in einigen Studien in vivo sowie in vitro untersucht und mit herkömmlichen externen Fixateuren verglichen.

Diese Studie zeigte eine in einigen Messungen signifikante Überlegenheit des SIAF hinsichtlich der Rotationssteifigkeit und ähnliche Ergebnisse bezüglich der Translationssteifigkeit, die erstmals mithilfe eines zyklischen Versuchaufbaus durchgeführt wurde. Die geringe Lockerungsrate in den klinischen Studien über SIAF unterstützt zudem die Vorstellung einer besseren Verankerung im Knochen. Die in vitro Daten deuten darauf hin, dass der SIAF ebenso klinisch eingesetzt werden kann, wenn der Fix. ext. in der Therapie von Beckenringverletzungen allein als ausreichend erachtet wird. Demzufolge könnte der Patient früher mobilisiert und so die Morbidität signifikant gesenkt werden.

SIAF sind jedoch bisher auch in bis zu 27\% der Fälle mit Irritationen des N. cutaneus femoralis lateralis sowie in bis zu $32 \%$ der Fälle mit heterotopen Ossifikationen assoziiert. Zusätzlich befinden sich Komponenten des SIAF in unmittelbarer Nähe des femoralen Gefäßbündels sowie der Harnblase, somit hängt der Erfolg auch maßgeblich von der Geschicklichkeit und Erfahrung des Operateurs ab. Der SIAF scheint trotz des o.g. Risikoprofils, als eine nützliche Anwendung in der Behandlung von Beckenringverletzungen, besonders bei Patienten mit abdominalen Begleitverletzungen oder Risikofaktoren für Pininfektionen. Dieses in vitro bestätigte Potential gilt es nun in vivo weiter zu erforschen und für den klinischen Alltag bei Überlegenheit zugänglich zu machen.

\section{Stephanie Simon}

Chirurgische Klinik und Poliklinik

Abt. für Unfall-, Hand- und Wiederherstellungschirurgie

Universitätsmedizin Rostock

Stephanie.Simon@uni-rostock.de 